\title{
Hemostasis management and therapeutic plasma exchange: Results of a practice survey
}

\author{
Nicole D. Zantek ${ }^{1}$ (1) | Leonard I. Boral ${ }^{2}$ | Yanhua Li $^{3}$ | Chisa Yamada ${ }^{4}$ (b) | Annika M. Svensson ${ }^{5}$ | \\ Jason E. Crane ${ }^{6}$ | Roy E. Smith ${ }^{7}$ | Monica B. Pagano ${ }^{8}$ | Marian A. Rollins-Raval $^{9}$ | \\ Amy E. Schmidt ${ }^{10}$ | Edward C. C. Wong ${ }^{11,12}$ | Yanyun $\mathrm{Wu}^{13}$ (b)
}

${ }^{1}$ Department of Laboratory Medicine and

Pathology, University of Minnesota, Minneapolis,

Minnesota

${ }^{2}$ Department of Pathology and Laboratory

Medicine, University of Kentucky, Lexington,

Kentucky

${ }^{3}$ Department of Pathology and Laboratory

Medicine, New York University, New York,

New York

${ }^{4}$ Department of Pathology, University of

Michigan, Ann Arbor, Michigan

${ }^{5}$ Consultant, Denver, Colorado

${ }^{6}$ LifeSource Blood Center, Rosemont, Illinois

${ }^{7}$ Hematology/Oncology Division, University of

Pittsburgh Medical Center Presbyterian-Shadyside

Hospital, Pittsburgh, Pennsylvania

${ }^{8}$ Department of Laboratory Medicine, University

of Washington, Seattle, Western Australia

${ }^{9}$ Department of Pathology and Laboratory

Medicine, University of North Carolina, Chapel

Hill, North Carolina

${ }^{10}$ Department of Pathology and Laboratory

Medicine, University of Rochester Medical Center,

Rochester, New York

${ }^{11}$ Department of Coagulation, Quest Diagnostics

Nichols Institute, Virginia

${ }^{12}$ Departments of Pediatrics and Pathology,

George Washington School of Medicine and

Health Sciences, Washington, DC

${ }^{13}$ Bloodworks, Seattle, Western Australia

Correspondence

Nicole D. Zantek, Department of Laboratory

Medicine and Pathology, University of Minnesota, 420 Delaware Street SE, MMC 609, Minneapolis, MN 55455.

Email: zant0005@umn.edu

\begin{abstract}
Background: Patients undergoing therapeutic plasma exchange (TPE) may present with risks for hemorrhage or thrombosis. Use of replacement fluids devoid of coagulation factors will decrease factor levels and platelet levels. There are no established guidelines for hemostasis management in these situations.

Materials and methods: A survey to evaluate current hemostasis management practice during TPE was conducted using online survey software. One response per institution was analyzed based on a hierarchical algorithm, excluding membrane filtration users, resulting in a maximum of 107 respondents. Descriptive analysis was performed with results reported as the number and frequency (\%) of respondents to each question.

Results: Apheresis Medicine physicians, alone (59.4\%) or jointly with the requesting provider (29.2\%), choose the replacement fluid. Based on a theoretical patient case receiving five TPEs approximately every other day, the percent of respondents who would use albumin with or without normal saline was $94.7 \%$ with no history of a bleeding or clotting disorder, $1.1 \%$ with active bleeding, and $8.8 \%$ with hypofibrinogenemia $(<100 \mathrm{mg} / \mathrm{dL})$ due to recent TPE. More respondents would use albumin with or without normal saline for replacement fluid when a minor invasive procedure (49.5\%) vs a major surgery (8.9\%) was performed 1 day before TPE. Replacement fluid selection varied among respondents for several other clinical conditions. The most frequent use for cryoprecipitate by respondents (14.3\%) was hypofibrinogenemia.

Conclusions: These survey results demonstrate wide interinstitutional variation in replacement fluid selection to manage hemostasis in patients undergoing TPE. Further studies are needed to guide optimal hemostasis management with TPE.
\end{abstract}

\section{KEYWORDS}

anticoagulation, apheresis, bleeding, coagulation, plasmapheresis

\section{1 | INTRODUCTION}

Apheresis is a procedure in which blood of the patient or donor is passed through a medical device which separates one or more components of blood and returns the remainder with or without extracorporeal treatment or replacement of the separated component. ${ }^{1}$ Therapeutic apheresis has become a widely used treatment or treatment alternative for many diseases or disease conditions, among which therapeutic plasma exchange (TPE) is the procedure performed most commonly 
in clinical practice. ${ }^{1,2} \mathrm{TPE}$ is a therapeutic apheresis procedure in which the plasma is removed and replaced with a solution such as colloid solution (eg, albumin and/or plasma) or a combination of crystalloid/colloid solution. TPE procedures performed with centrifugation based devices may remove pathologic substances (eg, toxins, autoantibodies, and paraproteins) as well as normal plasma constituents (eg, coagulation factors and natural anticoagulants). ${ }^{3-7}$ Circulating medications, such as anticoagulants, may also be removed. ${ }^{8-11}$ Because of the removal of coagulation factors and anticoagulants, it is a challenge in clinical apheresis medicine to maintain patients in a safe hemostasis status, which is further compounded in patients with an underlying risk for or ongoing hemorrhage or thrombosis.

Currently, there are no consensuses or national guidelines regarding hemostasis management in patients undergoing TPE treatments. To understand current practice, the Coagulation Subcommittee of the American Society for Apheresis (ASFA) Applications Committee conducted a survey regarding hemostasis management and TPE. This manuscript contains management practice data on the replacement fluid choice for patients undergoing TPE, while the laboratory testing practices are described in the associated article by Zantek et al. ${ }^{12}$ The aim of this study is to describe and characterize the current practice of replacement fluid selection for patients undergoing TPE.

\section{2 | METHOD}

A survey was developed by the members of the Coagulation Subcommittee of the ASFA Applications Committee to evaluate management of hemostasis in patients undergoing TPE. The development, implementation, and analysis of the survey is described in the associated article by Zantek et al. ${ }^{12}$ The data analyzed here represents the second portion of the survey. Because of decreasing numbers of responses through the progression of the survey, only those respondents that answered question 27 of 37 questions "What method of cell separation do you typically use for TPE" are included in the data set analyzed here. From the initial 167 responses, a total of 107 were included in these analyses. The reasons that 60 responses were excluded are as follows: 16 were duplicate responses, 24 were more than one response received from an institution, 15 did not answer question 27 of 37 (method of apheresis question), and 5 indicated they use membrane filtration or centrifugation and/or membrane filtration techniques.

\section{3 | RESULTS}

Demographic information is provided in Table 1. The respondents were mostly comprised of physicians (including physicians-in-training) and nurses and the procedures are mostly performed or overseen in the departments of Pathology and Hematology. Respondents who perform TPE on pediatric patients define a pediatric patient based on age (78.2\%) or weight (22.8\%). The most common age used by respondents to define a pediatric patient was 18 years (59.4\%). Weight-based definitions were varied and ranged from 5 to $50 \mathrm{~kg}$. The choice of replacement fluid to use for TPE procedures was made by the Apheresis Medicine physician (59.4\%), jointly by the Apheresis Medicine physician and requesting provider (29.3\%), the requesting provider (8.5\%), and other (2.8\%).

\section{1 | Routine practice at a responding center}

To understand routine practice at a center, respondents were asked how they manage a patient who presents for a series of five TPE approximately every other day. The most common procedural parameters were a 1.0 plasma volume (63.2\%) and use of anticoagulant citrate dextrose A for extracorporeal anticoagulation $(97.1 \%) .{ }^{12}$ The choice of replacement fluid was albumin, albumin and normal saline, plasma, albumin and plasma, and other by $47.7 \%, 12.2 \%, 3.7 \%$, $2.8 \%$, and $33.6 \%$ of respondents, respectively. Comments were provided by all 36 respondents who selected other for replacement fluid; no comments were entered by respondents who selected a specific replacement fluid. The comments from 27 of the 36 respondents included some form of the word "depend." Interpreting the 36 text comments, the choice of replacement fluid would depend on the diagnosis $(80.6 \%)$, laboratory values $(11.1 \%)$, and both diagnosis and laboratory values $(8.3 \%)$.

\section{2 | TPE with a potential bleeding risk}

When a patient presents for a series of five approximately every other day TPE with an associated potential bleeding risk, wide variation in the selection of replacement fluid was observed (Table 2). Similar to the case scenario used to decipher the respondent's routine practice, 27 respondents entered comments, many noting the replacement fluid choice would depend on the condition treated and other issues, such as results of laboratory studies and the specific patient clinical scenario. No respondent selected the same replacement fluids for all of the situations presented. However, 2 (2.1\%) respondents only selected replacement fluids containing some or all plasma and 3 (3.2\%) respondents only selected replacement fluids containing some or all plasma or cryoprecipitate or would chose to delay the procedure for the scenarios they provided a response.

\section{3 | Invasive procedures}

The replacement fluid selection was associated with the timing of minor invasive procedures and major surgery as shown in Tables 3 and 4. There was a trend after either a minor or major procedure to switch from a replacement fluid 
TABLE 1 Demographic information of survey respondents and apheresis programs

Question (number of respondents)

Number $(\%)^{\mathbf{b}}$

Position at institution $^{\mathrm{b}}(\mathrm{n}=107)^{\mathrm{c}}$

Physician (including physician in training-resident/fellow)

Director of apheresis service

Member of physician teaching staff

Nurse

Director of apheresis service

Member of nonphysician teaching staff

Other

Director of apheresis (did not indicate physician or nurse)

Type of institution $(\mathrm{n}=107)$

Academic medical center

Blood collection facility

Non-academic medical center

Contract provider of apheresis services

Outpatient facility that performs apheresis

Other

Number of beds for medical centers $(n=87)$

$>500$

100-500

$<100$

Apheresis procedures per year $(\mathrm{n}=105)$

$>700$

500-700

Departments which perform or oversee $\operatorname{TPE}^{\mathrm{b}}(\mathrm{n}=106)^{\mathrm{d}}$

Pathology

Hematology

Nephrology

Blood collection facility

Neurology

Performed by outside source but overseen by pathology

Performed by outside source but overseen by neurology

Performed by outside source but overseen by hematology

Performed by outside source but overseen by nephrology

Performed by outside source but overseen by rheumatology

Performed by outside source but overseen by medicine

Other

Physician medical specialty $(\mathrm{n}=95)$

Pathology

Hematology

Nephrology

Pediatrics

Medicine 
TABLE 1 (Continued)

\begin{tabular}{|c|c|}
\hline Question (number of respondents) ${ }^{\mathbf{a}}$ & Number $(\%)^{b}$ \\
\hline I am not a physician & $19(20.0)$ \\
\hline \multicolumn{2}{|c|}{ Perform TPE procedures on children $(n=107)$} \\
\hline No & $32(29.9)$ \\
\hline Yes, children only & $6(5.6)$ \\
\hline
\end{tabular}

${ }^{a}$ For some questions respondents were requested to check all that apply and the total number of responses is greater than the number of respondents.

${ }^{\mathrm{b}}$ For questions that permitted more than one response, the percent is based on the number of respondents to the question.

${ }^{\mathrm{c}}$ Total number of responses $=167$.

${ }^{\mathrm{d}}$ Total number of responses $=173$.

containing coagulation factors to albumin with or without normal saline. Some respondents entered free text comments to describe their practice, which indicated the choice of replacement fluid would depend on screening coagulation laboratory test results, such as the prothrombin time (PT), international normalized ratio (INR), partial thromboplastin time (PTT), and fibrinogen, or liver function test results.

\section{4 | Kidney biopsy}

The replacement fluid choice after kidney biopsy is shown in Supporting Information Table S1. The majority of respondents $(65.5 \%)$ indicated their routine practice for use of plasma in the replacement fluid after kidney biopsy depended on the number of days, hours, or procedure numbers since the biopsy, while $19.0 \%$ only use plasma when there is bleeding. When plasma is used up to specific days postbiopsy, 27 respondents indicated the number of days postbiopsy that plasma would be used is as follows (n, \%): 1 day $(5,18.5 \%), 2$ days $(9,33.3 \%), 3$ days $(4,14.8 \%)$, 3-5 days $(1,3.7 \%), 5$ days $(3,11.1 \%), 14$ days
(1, 3.7\%),15 days $(1,3.7 \%)$, and other $(3,11.1 \%)$. When plasma is used up to specific hours postbiopsy, four respondents provided a comment: one indicated plasma would be used up to 24-48 hours, one indicated up to 48 hours, and two indicated up to 72 hours. When plasma is used up to a specific number of procedures, eight respondents provided a comment: two indicated up to three TPE, one indicated up to seven TPE, and five provided a comment that did not give a specific procedure number. Additionally, a few respondents stated the amount of plasma they would use, which ranged from $30 \%$ to $100 \%$ of plasma replacement volume, and one respondent indicated a specific volume of 4 units of plasma would be used as part of the replacement fluid.

\section{5 | Antiplatelet and anticoagulant therapy}

Antiplatelet therapy use is common among the general population and may be used by patients undergoing TPE. The replacement fluid selection in the setting of an inherited or acquired platelet disorder is described in Table 2.The survey showed 8 out of 81 (9.9\%) respondents would stop ongoing

TABLE 2 Replacement fluid selection for therapeutic plasma exchange (TPE) in patients with an associated clinical situation and scheduled to receive five TPE approximately every other day

\begin{tabular}{|c|c|c|c|c|c|c|}
\hline \multirow[b]{2}{*}{ Associated situation } & \multicolumn{5}{|c|}{ Replacement fluid } & \multirow{2}{*}{$\begin{array}{l}\text { Delay } \\
\text { procedure } \\
\text { n }(\%)\end{array}$} \\
\hline & $\begin{array}{l}\text { Albumin } \\
\text { n (\%) }\end{array}$ & $\begin{array}{l}\text { Albumin and } \\
\text { normal saline } \mathbf{n}(\%)\end{array}$ & $\begin{array}{l}\text { Albumin and } \\
\text { plasma n (\%) }\end{array}$ & $\begin{array}{l}\text { Albumin and } \\
\text { CRYO n (\%) }\end{array}$ & $\begin{array}{l}\text { Plasma } \\
\text { n }(\%)\end{array}$ & \\
\hline No history of a bleeding or clotting disorder $(\mathrm{n}=94)$ & $71(75.5)$ & $18(19.1)$ & $4(4.3)$ & $0(0.0)$ & $1(1.1)$ & $0(0.0)$ \\
\hline Active bleeding $(\mathrm{n}=94)$ & $0(0.0)$ & $1(1.1)$ & $35(37.2)$ & $0(0.0)$ & $41(43.6)$ & $17(18.1)$ \\
\hline $\begin{array}{l}\text { Bleeding disorder due to coagulation factor deficiency (eg, } \\
\text { hemophilia A, B, or C; von Willebrand disease) }(\mathrm{n}=90)\end{array}$ & $9(10.0)$ & $0(0.0)$ & $35(38.9)$ & $4(4.4)$ & $35(38.9)$ & $7(7.8)$ \\
\hline Therapeutic on continuous IV unfractionated heparin $(n=89)$ & $31(34.8)$ & $9(10.1)$ & $28(31.5)$ & $2(2.3)$ & $13(14.6)$ & $6(6.7)$ \\
\hline $\begin{array}{l}\text { Supratherapeutic on continuous IV unfractionated heparin } \\
\qquad(\mathrm{n}=88)\end{array}$ & $19(21.6)$ & $7(8.0)$ & $24(27.3)$ & $2(2.3)$ & $22(25.0)$ & $14(15.9)$ \\
\hline $\begin{array}{l}\text { Therapeutic on low molecular weight heparin (eg, enoxaparin) } \\
(\mathrm{n}=87)\end{array}$ & $45(51.7)$ & $12(13.8)$ & $22(25.3)$ & $3(3.5)$ & $5(5.8)$ & $0(0.0)$ \\
\hline Supratherapeutic (INR 4.0) on warfarin $(\mathrm{n}=85)$ & $13(15.3)$ & $4(4.7)$ & $39(45.9)$ & $2(2.4)$ & $16(18.8)$ & $11(12.9)$ \\
\hline Liver disease with a mildly elevated INR $(1.5-2.0)(\mathrm{n}=87)$ & $29(33.3)$ & $4(4.6)$ & $43(49.4)$ & $1(1.2)$ & $10(11.5)$ & $0(0.0)$ \\
\hline
\end{tabular}

Abbreviations: CRYO, cryoprecipitate; INR, international normalized ratio; n, number of respondents. 
TABLE 3 Timing of minor invasive procedure and replacement fluid used for therapeutic plasma exchange

\begin{tabular}{|c|c|c|c|c|}
\hline & $\begin{array}{l}\text { In the morning of } \\
\text { TPE } n(\%)(n=91)\end{array}$ & $\begin{array}{l}1 \text { day before TPE } \\
\text { n }(\%)(n=91)\end{array}$ & $\begin{array}{l}2 \text { days before TPE } \\
\mathbf{n}(\%)(\mathrm{n}=91)\end{array}$ & $\begin{array}{l}2 \text { days before TPE } \\
\text { with bleeding } n(\%)(n=90)\end{array}$ \\
\hline Albumin & $16(17.6)$ & $34(37.4)$ & $52(57.1)$ & $1(1.1)$ \\
\hline Albumin and NS & $7(7.7)$ & $11(12.1)$ & $17(18.7)$ & $3(3.3)$ \\
\hline Albumin and plasma & $41(45.0)$ & $38(41.8)$ & $16(17.6)$ & $49(54.4)$ \\
\hline Albumin and CRYO & $1(1.1)$ & $0(0)$ & $1(1.1)$ & $0(0)$ \\
\hline Plasma & $10(11.0)$ & $5(5.5)$ & $5(5.5)$ & $26(28.9)$ \\
\hline Delay procedure & $16(17.6)$ & $3(3.3)$ & $0(0)$ & $11(12.2)$ \\
\hline
\end{tabular}

Abbreviations: CRYO, cryoprecipitate; n, number of respondents; NS, normal saline; TPE, therapeutic plasma exchange.

aspirin therapy, an inhibitor of prostaglandin cyclooxygenase-1, during a series of TPE, while 13 out of $80(16.3 \%)$ respondents would stop clopidogrel, an inhibitor of the $\mathrm{P}^{2} \mathrm{Y}_{12}$ class of the adenosine diphosphate receptors on platelets. When the patient is on both aspirin and clopidogrel $(n=79$ respondents), 9 (11.4\%) respondents indicated both medications would be stopped, while 3 (3.9\%) would stop aspirin only, and 7 (8.9\%) would stop clopidogrel only.

Anticoagulants are frequently encountered in patients undergoing TPE. See Table 2 for TPE replacement fluid choices in the settings of unfractionated heparin, low molecular weight heparin, and warfarin. Since 2010, there have been a number of new anticoagulants approved by the FDA. Respondents indicated they have performed TPE on patients receiving these therapies: 20 out of 76 (26.3\%) respondents for oral direct factor Xa inhibitor (eg, rivaroxaban, apixaban, edoxaban, and betrixaban); 16 out of $73(21.9 \%)$ respondents for oral direct thrombin inhibitor (eg, dabigatran); and 20 out of $72(27.8 \%)$ respondents for intravenous direct thrombin inhibitor (eg, argatroban, bivalirudin, and lepirudin). Respondents reported encountering patients on these medications in the context of left ventricular assist device (LVAD), extracorporeal membrane oxygenation (ECMO), heparin induced thrombocytopenia (HIT), trauma, transplant rejection, Blalock-Taussig shunt, and antiphospholipid antibody syndrome (APAS). Many respondents entered comments regarding management during TPE in patients on anti-Xa or direct thrombin inhibitors. Some respondents stated a concern for thrombosis in patients on these medications outweighed concerns of bleeding, so that they would not use a reversal agent or hold the procedure for 24 hours after the last dose. Replacement fluid selection varied from no change in practice to including some or all plasma. Some respondents indicated they would hold the medications while others would not.

\section{4 | DISCUSSION}

The selection of replacement fluid is a critical management decision for patients undergoing TPE using centrifugation based apheresis instruments. Based on available evidence, the ASFA guidelines for therapeutic apheresis suggest replacement fluids, but additional situational conditions may influence this choice. ${ }^{1}$ A survey was conducted to assess the current practice and the variation among apheresis providers.

To quote the respondents, the choice of replacement fluid "depends" on several factors including the diagnosis treated, laboratory studies, and other conditions present. Respondents were asked to describe how they would manage a case patient that is undergoing a series of five TPE approximately every other day. Our intent was to not bias the choice of replacement fluid by giving a specific indication for the TPE; however, this likely resulted in respondents considering very different patient scenarios. When more clarity was given regarding the patient, that is, that the patient had no history of a bleeding or clotting disorders, $94.7 \%$ of respondents would use albumin with or without normal saline. The remaining five respondents would use some or all plasma or delay the procedure for all of the clinical situations for which they provided a response. In retrospect it may have been ideal to include two specific cases where the replacement fluid choice suggested by the ASFA

TABLE 4 Timing of major surgery and replacement fluid used for therapeutic plasma exchange

\begin{tabular}{|c|c|c|c|c|c|}
\hline & $\begin{array}{l}2 \text { days after } \\
\text { TPE } n(\%)(n=90)\end{array}$ & $\begin{array}{l}1 \text { day after TPE } \\
\mathrm{n}(\%)(\mathrm{n}=91)\end{array}$ & $\begin{array}{l}1 \text { day before TPE } \\
\text { n }(\%)(n=90)\end{array}$ & $\begin{array}{l}3 \text { days before TPE } \\
\mathrm{n}(\%)(\mathrm{n}=90)\end{array}$ & $\begin{array}{l}1 \text { week before TPE } \\
\text { n }(\%)(\mathbf{n}=90)\end{array}$ \\
\hline Albumin & $35(38.9)$ & $10(11.0)$ & $5(5.6)$ & $24(26.7)$ & $53(58.9)$ \\
\hline Albumin and NS & $11(12.2)$ & $7(7.7)$ & $3(3.3)$ & $12(13.3)$ & $16(17.8)$ \\
\hline Albumin and plasma & $31(34.4)$ & $44(48.4)$ & $42(46.7)$ & $36(40.0)$ & $17(18.9)$ \\
\hline Albumin and CRYO & $1(1.1)$ & $1(1.1)$ & $1(1.1)$ & $0(0)$ & $0(0)$ \\
\hline Plasma & $11(12.2)$ & $24(26.4)$ & $31(34.4)$ & $16(17.8)$ & $2(2.2)$ \\
\hline
\end{tabular}

Abbreviations: CRYO, cryoprecipitate; n, number of respondents; NS, normal saline; TPE, therapeutic plasma exchange. 
guidelines is suggested to be albumin (eg, myasthenia gravis) and plasma (eg, thrombotic thrombocytopenic purpura). ${ }^{1}$ The ASFA recommendations of the Choosing Wisely initiative of the ABIM Foundation recommends "Do not routinely use plasma as replacement fluid for therapeutic plasma exchange unless there is a clear indication to replete a plasma component". ${ }^{13}$

Cryoprecipitate was used by a small number of respondents. The most frequent use was for fibrinogen replacement in patients with hypofibrinogenemia because of recent TPE. Small numbers of respondents (1 to 4) would use cryoprecipitate in other situations. In patients with a coagulation factor deficiency, cryoprecipitate would also be valuable for factor VIII, von Willebrand factor, and factor XIII replacement, though it is not clear that this was the motivation behind the selection.

Use of coagulation factor deficient replacement fluids for TPE proximate to an invasive procedure may increase the risk for bleeding because of the decrease in coagulation factors. Thus, some apheresis providers may use plasma or cryoprecipitate to potentially mitigate this risk. This practice varies among institutions. For example following kidney biopsy, 5 of $84(6.0 \%)$ respondents indicate they would use plasma for up to five or more days after the biopsy, while 13 of 84 (15.5\%) of respondents do not routinely use plasma. With other minor invasive procedures, $25.3 \%$ would use albumin with or without normal saline even if the invasive procedure is performed in the morning of the TPE. When the invasive procedure is major surgery, more respondents would include some coagulation factor replacement, but $8.9 \%$ would perform the TPE with albumin with or without normal saline 1 day after a major surgery. When considering minor vs major procedures, one limitation of our study was the different time periods queried between the two scenarios. Additionally, the data on the use of plasma after a kidney biopsy may have been clearer to interpret if we had presented the case patient at multiple days after the biopsy and inquired what replacement fluid they would use. However, this would have added several more questions to the end of an already long survey.

There is very limited data published on the anticoagulant management in patients undergoing TPE. ${ }^{14-22}$ As illustrated in Table 2, replacement fluid containing some or all plasma or cryoprecipitate would be used by $34.5 \%-67.1 \%$ of respondents with the common anticoagulants unfractionated heparin, low molecular weight heparin, and warfarin. Several respondents would use plasma as the replacement fluid (solely $6.9 \%$ or with albumin $35.6 \%$ ) in a patient who is therapeutic (INR 2.5) on warfarin, a vitamin $\mathrm{K}$ antagonist (VKA). Plasma transfusion can be used as a reversal agent for VKAs. ${ }^{23-25}$ The use of all plasma would be expected to result in significant reversal of the patient's anticoagulation. Although approximately a quarter of respondents have performed TPE on patients on oral (eg, dabigatran) or intravenous (eg, lepirudin, argatroban, and bivalidrudin) direct thrombin inhibitor or an oral direct anti-Xa inhibitor (eg, rivaroxaban, apixaban, edoxaban, betrixaban), the impact of TPE on patients on anticoagulants is not entirely clear. The procedure may remove the anticoagulant medications as well as the specific anticoagulant targets. There is limited information on medication removal with TPE, but several factors influence this including protein binding, volume of distribution, dose, timing since last dose; volume of TPE, length of the TPE procedure, successive TPE procedures, and potentially the replacement fluid. ${ }^{8-11}$ Further investigation is needed to establish the best practice with regard to anticoagulants and antiplatelet therapy in the setting of TPE.

There are several other limitations to this study. Because of the length of the survey, there was a decrease in the number of respondents throughout the course of the survey. It is unknown if the practices of the respondents who did not complete the survey would be different from other respondents. Because of the small numbers, data from respondents using membrane filtration were excluded from analyses. The majority of respondents were from the United States of America. The number of TPE procedures performed by the respondents is not known as we inquired about total number of apheresis procedures rather than the specific number of TPE. An apheresis provider's background, training, and experiences likely contribute to the choice of replacement fluid; however, the survey did not capture adequate information to analyze these factors.

\section{5 | CONCLUSIONS}

The selection of the replacement fluid for TPE depends on many variables, including the diagnosis for performing the procedure, concurrent clinical situations, and laboratory studies. There is little evidence to guide the adjustment of the replacement fluid content, which likely contributed to the wide variation in practices seen in this survey. Future research study is needed to establish best practice.

\section{ACKNOWLEDGMENTS}

The authors would like to acknowledge the respondents who participated in our survey, the members of the ASFA Clinical Applications Committee who provided feedback during development and beta testing of the survey, and the ASFA head office staff for assistance with distributing the survey and administrative support throughout the project.

\section{DISCLOSURE OF INTEREST}

NDZ: Research funding: Octapharma, Terumo BCT, Bayer HealthCare, Verax Biomedical; Financial interest: Endo International PLC, Boston Scientific. MBP: Research 
funding: Terumo BCT. MAR: Advisory Boards: Bayer and NovoNordisk. ECCW: Consultant: Comprehensive Care Services. YW: Consultant: Verax Biomedical. The other authors have no conflict of interest to disclose.

\section{ORCID}

Nicole D. Zantek (D) http://orcid.org/0000-0001-5776-6400

Chisa Yamada (D) http://orcid.org/0000-0002-1968-2385

Yanyun Wu (D) http://orcid.org/0000-0001-8410-4349

\section{REFERENCES}

1. Schwartz J, Padmanabhan A, Aqui N, et al. Guidelines on the use of therapeutic apheresis in clinical practice-evidence-based approach from the writing Committee of the American Society for apheresis: the seventh special issue. J Clin Apher. 2016;31(3):149-162.

2. Winters JL. Plasma exchange: concepts, mechanisms, and an overview of the American Society for Apheresis guidelines. Hematology Am Soc Hematol Educ Program. 2012;2012:7-12.

3. Witt V, Pichler H, Beiglboeck E, Kursten F, Weidner L. Changes in hemostasis caused by different replacement fluids and outcome in therapeutic plasma exchange in pediatric patients in a retrospective single center study. Transfus Apher Sci. 2017;56(1):59-65.

4. Tek I, Arslan O, Arat M, Ozcan M, Akdag B, Ilhan O. Effects of replacement fluids on coagulation system used for therapeutic plasma exchange. Transfus Apher Sci. 2003;28(1):3-7.

5. Volkin RL, Starz TW, Winkelstein A, et al. Changes in coagulation factors, complement, immunoglobulins, and immune complex concentrations with plasma exchange. Transfusion. 1982;22(1):54-58.

6. Orlin JB, Berkman EM. Partial plasma exchange using albumin replacement: removal and recovery of normal plasma constituents. Blood. 1980;56(6): 1055-1059.

7. Wood L, Jacobs P. The effect of serial therapeutic plasmapheresis on platelet count, coagulation factors, plasma immunoglobulin, and complement levels. J Clin Apher. 1986;3(2):124-128.

8. Ibrahim RB, Balogun RA. Medications in patients treated with therapeutic plasma exchange: prescription dosage, timing, and drug overdose. Semin Dial. 2012;25(2):176-189.

9. Ibrahim RB, Balogun RA. Medications and therapeutic apheresis procedures: are we doing our best? J Clin Apher. 2013;28(1):73-77.

10. Ibrahim RB, Liu C, Cronin SM, et al. Drug removal by plasmapheresis: an evidence-based review. Pharmacotherapy. 2007;27(11):15291549.

11. Kale-Pradhan PB, Woo MH. A review of the effects of plasmapheresis on drug clearance. Pharmacotherapy. 1997;17(4):684-695.

12. Zantek ND, Pagano MB, Rollins-Raval MA, et al. Hemostasis testing and therapeutic plasma exchange: results of a practice survey. J Clin Apher.

13. ABIM Foundation. Choosing Wisely http://www.choosingwisely.org/ clinician-lists. Accessed May 8, 2018.
14. Kaplan A, Raut P, Totoe G, Morgan S, Zantek ND. Management of systemic unfractionated heparin anticoagulation during therapeutic plasma exchange. J Clin Apher. 2016;31(6):507-515.

15. Zantek ND, Morgan S, Zantek PF, Mair DC, Bowman RJ, Aysola A. Effect of therapeutic plasma exchange on coagulation parameters in patients on warfarin. J Clin Apher. 2014;29(2):75-82.

16. Keller AJ, Chirnside A, Urbaniak SJ. Coagulation abnormalities produced by plasma exchange on the cell separator with special reference to fibrinogen and platelet levels. Br J Haematol. 1979;42(4):593-603.

17. Qui J, Zantek ND, Kaplan LI, et al. Management of an apheresis patient with a mechanical aortic valve on heparin and warfarin anticoagulation. $J$ Clin Apher. 2013;28.

18. Rahawi KW, Higgins KL, Noda C, Stultz JS. Effect of plasmapheresis on the anti-factor Xa activity of enoxaparin in an obese adolescent patient. Pharmacotherapy. 2017;37(4):e16-e20.

19. Unal S, Bayrakci B, Yasar U, Karagoz T. Successful treatment of propafenone, digoxin and warfarin overdosage with plasma exchange therapy and rifampicin. Clin Drug Investig. 2007;27(7):505-508.

20. Lam WW, Reyes MA, Seger JJ. Plasma exchange for urgent Apixaban reversal in a case of hemorrhagic tamponade after pacemaker implantation. Tex Heart Inst J. 2015;42(4):377-380.

21. Preston TJ, Dalton HJ, Nicol KK, Ferrall BR, Miller JC, Hayes D Jr. Plasma exchange on venovenous extracorporeal membrane oxygenation with bivalirudin anticoagulation. World J Pediatr Congenit Heart Surg. 2015;6(1):119-122.

22. Shunkwiler SM, Pham HP, Wool G, et al. The management of anticoagulation in patients undergoing therapeutic plasma exchange: a concise review. $J$ Clin Apher. 2017.

23. Ageno W, Gallus AS, Wittkowsky A, Crowther M, Hylek EM, Palareti G. Oral anticoagulant therapy: antithrombotic therapy and prevention of thrombosis, 9th ed: American College of Chest Physicians Evidence-Based Clinical Practice Guidelines. Chest. 2012;141(2 Suppl):e44S-e88S.

24. Holbrook A, Schulman S, Witt DM, et al. Evidence-based management of anticoagulant therapy: antithrombotic therapy and prevention of thrombosis, 9th ed: American College of Chest Physicians Evidence-Based Clinical Practice Guidelines. Chest. 2012;141(2 Suppl):e152S-e184S.

25. Tomaselli GF, Mahaffey KW, Cuker A, et al. 2017 ACC expert consensus decision pathway on Management of Bleeding in patients on oral anticoagulants: a report of the American College of Cardiology Task Force on expert consensus decision pathways. J Am Coll Cardiol. 2017;70(24):3042-3067.

\section{SUPPORTING INFORMATION}

Additional supporting information may be found online in the Supporting Information section at the end of the article.

How to cite this article: Zantek ND, Boral LI, Li Y, et al. Hemostasis management and therapeutic plasma exchange: Results of a practice survey. J Clin Apher. 2018;33:604-610. https://doi.org/10.1002/jca.21653 